\title{
Effect of migration in a diffusion model for template coexistence in protocells
}

\author{
José F. Fontanari · Maurizio Serva
}

Received: date / Accepted: date

\begin{abstract}
The compartmentalization of distinct templates in protocells and the exchange of templates between them (migration) are key elements of a modern scenario for prebiotic evolution. Here we use the diffusion approximation of population genetics to study analytically the steady-state properties of such prebiotic scenario. The coexistence of distinct template types inside a protocell is achieved by a selective pressure at the protocell level (group selection) favoring protocells with a mixed template composition. In the degenerate case, where the templates have the same replication rate, we find that a vanishingly small migration rate suffices to eliminate the segregation effect of random drift and so to promote coexistence. In the non-degenerate case, a small migration rate greatly boosts coexistence as compared with the situation where there is no migration. However, increase of the migration rate beyond a critical value leads to the complete dominance of the more efficient template type (homogeneous regime). In this case, we find a continuous phase transition separating the homogeneous and the coexistence regimes, with the order parameter vanishing linearly with the distance to the transition point.
\end{abstract}

Keywords group selection · diffusion approximation · prebiotic evolution

Mathematics Subject Classification (2000) 92D15 -35Q92 $\cdot 82 \mathrm{~B} 27$

\section{Introduction}

The coexistence of competing selfish individuals is an ubiquitous issue in the study of systems described by the modern Darwinian paradigm, known as the "Evolutionary Synthesis" (Maynard Smith and Szathmáry, 1995, Mayr, 2001, 2002, 2004, Nowak and Sigmund,

\section{J. F. Fontanari}

Instituto de Física de São Carlos, Universidade de São Paulo, Caixa Postal 369, 13560-970 São Carlos SP, Brazil

E-mail: fontanari@ifsc.usp.br

M. Serva

Departamento de Biofísica e Farmacologia, Universidade Federal do Rio Grande do Norte, 59072-970 Natal, RN, Brazil

On leave of absence from Dipartimento di Ingegneria e Scienze dell'Informazione e Matematica, Università dell' Aquila, I-67010 Coppito, L'Aquila, Italy. 
2004). In the context of prebiotic or chemical evolution, this matter surfaced with the observation by Eigen (1971) that, due to the finite fidelity of replication, the information content of a single self-replicating macromolecule (a template for short) is far too few to permit the coding of macromolecules with any functional complexity. A way out of this difficulty, so-called the information crisis of prebiotic evolution, is to assume the information is distributed among a number of distinct template types and enforce cyclic cooperative interactions among them - the hypercycle - to guarantee coexistence (Eigen and Schuster 1978, Eigen et al. 1980).

Alternatively, coexistence between distinct template types can be achieved by confining the templates in packages or protocells and requiring that the survival or the reproduction chances of a protocell be dependent on its template composition (Bresch et al. 1980, Niesert et al. 1981; Szathmáry and Demeter. 1987). The study of this two-level selection problem can be carried out by introducing minor changes on the mathematical models developed to address the efficiency of group selection to maintain an altruistic trait (Eshel, 1972, Aoki. 1982, Donato et al. (1997). In particular, in a recent paper we have used a diffusion model of group selection (Kimura 1983) to study analytically the conditions for the coexistence of two template types which differ on their replication rates (Fontanari and Serva 2013). However, that study left out a crucial characteristic of the primitive protocell populations, namely, the elevated exchange flux of templates among protocells, known as lateral or horizontal gene transfer. In fact, the acceptance of the operation of this process in the early history of microbial life has wiped out completely the familiar Darwinian notion of a universal ancestor (Woese, 1998, Doolittle, 2000).

Here we model the process of template swapping among protocells by the classic migration process of Wright's island model (Wright, 1951). We find that introduction of migration renders the evolutionary process ergodic in the sense that the steady state does not depend on the initial set-up of the population. In addition, migration allows a steady-state solution corresponding to protocells carrying both template types (coexistence regime) or a solution where the more efficient template type is fixed in all protocells (homogeneous regime). There is a smooth transition between these two regimes provided that the two template types exhibit distinct replication rates. In the degenerate case, where the template types have identical replication efficiencies, only the coexistence regime is stable. This contrasts with the results obtained in the absence of migration, for which there is a non-ergodic segregation regime characterized by a mixture of two types of protocells, each type carrying solely one of the template types (Fontanari and Serva, 2013).

The remainder of the paper is organized as follows. In Sec. 2 we describe the three evolutionary processes - template competition, migration and intercell competition - that comprise the dynamics of our two-level selection model, and derive the partial differential equation that governs the time evolution of the fraction of protocells carrying a given template composition. Sec. 3 is devoted to the numerical and analytical study of the steady-state solutions of that equation. In particular, our numerical approach relies on the interpretation of the steady-state ordinary differential equation as an eigenvalue problem whose eigenvalue corresponds to the mean group selection pressure. Our concluding remarks are presented in Sec.4. In Appendix A we present the analytical calculation of the probability that a template type fixates in a given protocell in the non-ergodic segregation regime for the case migration is not allowed. This calculation generalizes that presented in Fontanari and Serva (2013) by taking into account the different replication efficiencies of the template types. 


\section{The model}

Following Kimura (1983), we consider a hypothetical population divided into an infinite number of competing protocells, each of which containing exactly $N$ templates. There are two types of templates which differ only by their replication efficiency: type 1 templates have a selective disadvantage $s$ relative to type 2 templates, where $s \geq 0$ is a parameter on the order of $1 / N$. More pointedly, type 1 templates are assigned fitness $1-s$ and type 2 templates fitness 1 . In addition, we assume that $N$ is large enough so that the frequency of type 1 templates within a protocell, denoted by $x$, can be viewed as a continuous variable in the interval $[0,1]$. Of course, the frequency of type 2 templates within the same protocell is $1-x$. The population is described by the fraction of protocells $\phi(x, t) \Delta x$ whose frequency of type 1 templates lies in the range $(x, x+\Delta x)$ at time $t$. Our goal is to determine how the probability density $\phi(x, t)$ is affected by the three evolutionary processes: individual template competition within a protocell, migration of templates between protocells and competition between protocells.

The template competition process within each protocell takes place according to the rules of the standard Wright-Fisher model of population genetics (Crow and Kimura, 1970). In particular, assuming that a protocell contains $j$ type 1 templates and $N-j$ type 2 templates, the probability that there will be exactly $i$ type 1 templates after template competition is given by the Wright-Fisher process

$$
r_{i j}=\left(\begin{array}{c}
N \\
i
\end{array}\right) w_{j}^{i}\left(1-w_{j}\right)^{N-i},
$$

where $w_{j}=j(1-s) /(N-j s)$ is the relative fitness of the subpopulation of type 1 templates in the protocell. To determine how this process affects the probability density $\phi(x, t)$ we resort to the diffusion approximation of population genetics (Crow and Kimura, 1970), which consists essentially on the calculation of the jump moments $\left\langle\left(x^{\prime}-x\right)\right\rangle_{r}$ and $\left\langle\left(x^{\prime}-x\right)^{2}\right\rangle_{r}$ where $x=j / N$ and $x^{\prime}=i / N$ are the frequencies of type 1 templates before and after template competition, respectively. Here $\langle\ldots\rangle_{r}$ stands for an average using the transition probability $r_{i j}$. These moments contribute to the drift and the diffusion terms of a forward Kolmogorovlike equation for $\phi(x, t)$. More pointedly, direct evaluation of the jump moments to first order in $1 / N$ using the transition probability $(1)$ yields

$$
\left\langle\left(x^{\prime}-x\right)\right\rangle_{r}=w_{j}-x \approx-s x(1-x)
$$

and

$$
\left\langle\left(x^{\prime}-x\right)^{2}\right\rangle_{r}=\frac{1}{N} w_{j}\left(1-w_{j}\right)+\left(w_{j}-x\right)^{2} \approx \frac{1}{N} x(1-x)
$$

where we have used that the fitness disadvantage $s$ of the type 1 templates is on the order of $1 / N$.

Migration follows Wright's island model (Wright 1951) that posits that $J$ templates of each protocell are replaced by migrants in the time interval $\Delta t$ and that the frequency of type 1 templates among the migrants is equal to the average frequency of type 1 templates in the entire protocell population, i.e., $\bar{x}=\int_{0}^{1} x \phi(x, t) d x$. The probability that a protocell with $j$ type 1 templates $(x=j / N)$ becomes a protocell with $i$ type 1 templates $\left(x^{\prime}=i / N\right)$ due to the migration process is then (Aoki 1982)

$$
m_{i j}=\sum_{k=k_{l}}^{k_{u}} \frac{\left(\begin{array}{l}
j \\
k
\end{array}\right)\left(\begin{array}{l}
N-j \\
J-k
\end{array}\right)}{\left(\begin{array}{l}
N \\
J
\end{array}\right)}\left(\begin{array}{c}
J \\
i-j+k
\end{array}\right) \bar{x}^{i-j+k}(1-\bar{x})^{J-i+j-k},
$$


where $k_{l}=\max (j-i, 0, J-N+j)$ and $k_{u}=\min (j, J-i+j, J)$. Here the hyper-geometric component yields the probability that exactly $k$ type 1 templates and $J-k$ type 2 templates are eliminated from the protocell to make room for the $J$ migrants, whereas the binomial part yields the probability that there are exactly $i-j+k$ type 1 templates among the $J$ migrants. After migration the number of type 1 templates in the protocell is given by the sum of the type 1 templates originally in the protocell $(j-k)$ and the number of type 1 templates among the migrants $(i-j+k)$. The first two jump moments are given by

$$
\left\langle\left(x^{\prime}-x\right)\right\rangle_{m}=m(\bar{x}-x)
$$

and

$$
\left\langle\left(x^{\prime}-x\right)^{2}\right\rangle_{m}=\frac{m}{N} \bar{x}(1-\bar{x})+m^{2}(\bar{x}-x)^{2}+\frac{m(1-m)}{N-1} x(1-x),
$$

where $\langle\ldots\rangle_{m}$ stands for an average using the transition probability $m_{i j}$ and $m=J / N$ is the fraction of the protocell population that is replaced by migrants. Assuming that $m$ is on the order of $1 / N$, i.e., that the number of migrants $J$ remains finite and limited when $N$ grows large, we can neglect the second jump moment which is $O\left(1 / N^{2}\right)$.

Finally, the competition between protocells is taken into account as follows. Denoting by $c(x)$ the selection coefficient of a protocell with a fraction $x$ of type 1 templates we have

$$
\phi(x, t+\Delta t)=[\phi(x, t)+c(x) \phi(x, t) \Delta t] \zeta,
$$

where $\zeta$ is such that $\int_{0}^{1} \phi(x, t+\Delta t) d x=1$, i.e, $\zeta=1 /[1+\bar{c}(t) \Delta t]$ with

$$
\bar{c}(t)=\int_{0}^{1} c(x) \phi(x, t) d x .
$$

Taking the limit $\Delta t \rightarrow 0$ we obtain the change in the fraction of protocells due to intercell selection, $\Delta \phi=[c(x)-\bar{c}(t)] \phi(x, t) \Delta t$.

Combining the changes in $\phi$ due to the three processes described above and introducing the rescaled variables $\tau=t / 2 N, S=2 N s \geq 0, M=2 N m \geq 0$ and $C(x)=2 N c(x) \geq 0$ we obtain (Kimura 1983)

$$
\frac{\partial}{\partial \tau} \phi(x, \tau)=\frac{\partial^{2}}{\partial x^{2}}[x(1-x) \phi(x, \tau)]-\frac{\partial}{\partial x}[b(x, \tau) \phi(x, \tau)]+[C(x)-\bar{C}(\tau)] \phi(x, \tau),
$$

where

$$
b(x, \tau)=-S x(1-x)-M[x-\bar{x}(\tau)]
$$

is the drift term,

$$
\bar{x}(\tau)=\int_{0}^{1} x \phi(x, \tau) d x
$$

is the mean number of type 1 templates in the protocell population, and

$$
\bar{C}(\tau)=\int_{0}^{1} C(x) \phi(x, \tau) d x
$$

is the mean group selection pressure. The constraint $\int_{0}^{1} \phi(x, \tau) d x=1$ holds for all times $\tau$.

We note that whereas the linear forward Kolmogorov equation is the standard output in the case of random drift and individual selection (Crow and Kimura. 1970), eq. (9) is nonlinear because of the presence of $\bar{x}(\tau)$ and $\bar{C}(\tau)$, which are associated to migration and group selection. In addition, the singularities (if any) of the solution of eq. 9) must be integrable so as to guarantee that it is normalizable for all times. 
Kimura's choice for the intercell selection coefficient, $C(x) \propto x$, aimed at exploring the efficiency of group selection to maintain an altruistic character - the type 1 template in that case - which has a selective disadvantage $s$ relative to its competitor but whose presence would boost the protocell reproduction rate, which increases linearly with the frequency of altruists inside it. We refer the reader to Ogura and Shimakura (1987) for a rigorous analysis of the linear intercell selection model introduced by Kimura (1983) and to Fontanari and Serva (2014) for the analysis of the nonlinear variant of Kimura's model. Here we consider the coexistence problem instead, which is more burdensome to group selection than the altruistic version, since the fixation of a template type through the effect of random drift, regardless of its selective advantage or disadvantage, acts against coexistence (Fontanari et al. 2006). According to the so-called metabolic model of template cooperation (Bresch et al. 1980, Niesert et al. 1981; Szathmáry and Demeter. 1987; Czárán and Szathmáry. 2000, Silvestre and Fontanari 2008), in order to favor coexistence we choose the intercell selection coefficient

$$
C(x)=C x(1-x)
$$

which is maximum for well-balanced protocells at which $x=1 / 2$. Here $C$ is a parameter on the order of 1 that measures the intensity of the group selection pressure towards coexistence. The idea behind eq. 13 is that the two functional template types coded for a small piece of a modular enzyme which then promoted protocell replication (Manrubia and Briones, 2007). Since the hookup of the replicase requires products from the two template types, its production rate is proportional to the concentration of the rare type, hence the requirement that $c(x)$ is maximized by well-balanced protocells.

The model has three parameters, namely, $S$ that measures the selective disadvantage of type 1 templates in the within cell competition process, $M$ that measures the strength of migration, and $C$ that measures the strength of the group selection pressure towards template coexistence. The scale of these parameters is given by the coefficient of the diffusion term which is set to 1 in eq. 9 .

\section{The steady-state solutions}

The steady-state protocell probability density $\phi=\phi(x)=\lim _{\tau \rightarrow \infty} \phi(x, \tau)$ satisfies

$$
\frac{d^{2}}{d x^{2}}[x(1-x) \phi]+\frac{d}{d x}[S x(1-x) \phi+M(x-\bar{x}) \phi]+[C x(1-x)-\bar{C}] \phi=0
$$

with $\bar{x}=\lim _{\tau \rightarrow \infty} \bar{x}(\tau), \bar{C}=\lim _{\tau \rightarrow \infty} \bar{C}(\tau)$, and $\int_{0}^{1} \phi(x) d x=1$.

For $M>0$, eq. 14 is satisfied both by $\phi=\delta(x)$ and $\phi=\delta(x-1)$, and it may also be satisfied by a regular function $\phi=\phi_{r}(x)$. By a regular solution of eq. 14 we intend a nonvanishing continuous function $\phi_{r}$ in the interval $[0,1]$ which is of class $C^{2}$ in $(0,1)$ where it satisfies (14). Since $\phi_{r}$ is a probability density we additionally restrict to normalizable functions, i.e. functions such that $\int_{0}^{1} \phi_{r}(x) d x$ is finite. We note that in the absence of the coexistence pressure $C=0$, the regular solution is missing (Crow and Kimura, 1970). However, one can easily verify that the migration term prohibits solutions which are combinations of the three possibilities (i.e., the deltas at $x=0$ and $x=1$ and the regular solution), since in that case eq. (14) would be violated in one of the two extremes, $x=0$ or $x=1$. Clearly, each possibility corresponds to protocell populations with distinct characteristics. In particular, $\phi=\delta(x)$ describes a population composed of type 2 templates only, $\phi=\delta(x-1)$ a 
population of type 1 templates only, and $\phi=\phi_{r}(x)$ describes the desired situation where the different templates cohabit a same protocell.

It is instructive to note that if a regular solution exists, then integration of eq. (14) over the interval $[0,1]$ yields

$$
\frac{d}{d x}\left(x \phi_{r}\right)-\left.M \bar{x} \phi_{r}\right|_{x=0}=0
$$

and

$$
\frac{d}{d x}\left[(1-x) \phi_{r}\right]-\left.M(1-\bar{x}) \phi_{r}\right|_{x=1}=0
$$

which imply that for $x$ close to 0 one has $\phi_{r} \sim x^{M \bar{x}-1}$, whereas for $x$ close to 1 one has $\phi_{r} \sim(1-x)^{M(1-\bar{x})-1}$. Hence, in spite of the fact that $\phi_{r}$ describes a regime of coexistence, this coexistence can be very unbalanced in the sense that the majority of the protocells may be populated by essentially a single template type. This unbalance is typical in the case $M<1$.

\subsection{Numerical analysis}

The steady-state solutions of a diffusion model of intergroup selection for the maintenance of an altruistic trait were obtained numerically by Kimura (1983) in the simple case of a linear group selection pressure $c(x) \propto x$, i.e., $\bar{C} \propto \bar{x}$. In that case eq. 14 exhibits only one non-local term and a straightforward self-consistent iterative approach yields the correct solution. In our case such a direct approach is doomed to failure, as it will become clear below.

Following Kimura (1983) we write the regular solution of eq. 14 in the form $\phi_{r}(x)=$ $\kappa \phi_{0}(x) \psi(x)$ where $\phi_{0}$ is the solution in the absence of group selection $(C=0)$ and for fixed $\bar{x} \neq 0,1$, namely,

$$
\phi_{0}=\exp (-S x) x^{M \bar{x}-1}(1-x)^{M(1-\bar{x})-1},
$$

and $\kappa$ is the normalization constant. Hence the equation for $\psi$ reads

$$
x(1-x) \frac{d^{2} \psi}{d x^{2}}-[S x(1-x)+M(x-\bar{x})] \frac{d \psi}{d x}+C x(1-x) \psi=\bar{C} \psi,
$$

which, as already pointed out, for fixed $\bar{x}$ can be viewed as an eigenvalue problem without boundary conditions that can be solved by requiring the regularity of $\psi(x)$ in $[0,1]$ only (Chalub and Souza 2009). In addition, according to the expected behavior of $\phi_{r}$ in the vicinities of $x=0$ and $x=1$ we can guarantee that $\psi$ is bounded at these extreme values. Of course, the attempt to solve eq. (18) numerically for an arbitrary value of $\bar{C}$ using, say the Runge-Kutta algorithm, results in divergences at the extremes, which ruins any selfconsistent iterative approach to solve this equation.

Next we define $\psi=\exp (y)$ and get the following nonlinear equation

$$
x(1-x)\left[y^{\prime \prime}+\left(y^{\prime}\right)^{2}\right]-[S x(1-x)+M(x-\bar{x})] y^{\prime}+C x(1-x)=\bar{C},
$$

where the primes indicate derivatives with respect to $x$. This is really a first order equation for $z \equiv y^{\prime}$,

$$
x(1-x)\left[z^{\prime}+z^{2}\right]-[S x(1-x)+M(x-\bar{x})] z+C x(1-x)=\bar{C},
$$


with

$$
z(0)=\frac{\bar{C}}{M \bar{x}}
$$

and

$$
z(1)=-\frac{\bar{C}}{M(1-\bar{x})} .
$$

At this stage the problem is ready for a numerical approach. For fixed $\bar{x}$ and $\bar{C}$ we solve eq. 20 by propagating the Runge-Kutta algorithm from $x=0$ to $x=1$ using the initial condition 21. Of course, the choice of an arbitrary value of $\bar{C}$ will not satisfy the boundary condition (22) so we adjust $\bar{C}$ in order that condition is satisfied. This is essentially an application of the well-known shooting method to solve boundary values problems (Press et al. 1992). Once this is achieved, we have solved the problem for a fixed $\bar{x}$. Explicitly, $y$ is obtained from

$$
y=\int_{0}^{x} z(\xi) d \xi
$$

where we have defined $y(0)=0$ (hence $\psi(0)=1$ ). This choice is inconsequential since the physical quantities are given by ratios of integrals involving $\psi=e^{y}$. In fact, we can then calculate $\bar{x}$,

$$
\bar{x}=\frac{\int_{0}^{1} x \phi_{0}(x) e^{y} d x}{\int_{0}^{1} \phi_{0}(x) e^{y} d x},
$$

return to eq. 20 and repeat the process until we reach the convergence for $\bar{x}$. In particular, we assume that convergence occurs whenever the change in $\bar{x}$ is less than $10^{-6}$ in two consecutive iteration steps. This iterative scheme is extremely efficient since it involves the numerical solution of a single first-order ordinary differential equation and the iteration over a single quantity only, namely $\bar{x}$.

In Fig. 1 we show the dependence of the eigenvalue $\bar{C}$ on the coexistence pressure parameter $C$ in the degenerate case $S=0$ and for a variety of values of the migration parameter. In this case, the symmetry of eqs. (14) and (18) with respect to the interchange of $x$ and $1-x$ yields $\bar{x}=1 / 2$ regardless of the values of $M$ and $C$. This is illustrated in Fig. 2 where the regular solution $\phi_{r}$ is shown for representative values of the migration parameter. Interestingly, the phase transition between the coexistence $(\bar{C}>0)$ and the segregation $(\bar{C}=0)$ phases that takes place at $C=\pi^{2}$ for $M=0$ and $S=0$ (Fontanari and Serva, 2013) disappears altogether when the process of migration is included in the model. The segregation phase, which is characterized by a well-balanced mixture of protocells composed of either type 1 or type 2 templates, is eliminated in this case. Hence, in the degenerate case where there is no selective advantage at the template level $(S=0)$, migration promotes coexistence (see Fig. 2.

The scenario becomes more interesting when the replication rates of the template types are allowed to differ, as illustrated in Figs. 3 and 4 for $S=1$. The first noteworthy result exhibited in these figures is the appearance of a phase transition separating the homogeneous regime dominated by the more efficient template type and characterized by $\bar{x}=\bar{C}=0$, from the coexistence regime, $\bar{C}>0$. We note that $\bar{x}>0$ does not imply coexistence, since this condition holds true in the segregating phase that exists for $M=0$ and is characterized by an unbalanced mixture of delta functions at the extremes $x=0$ and $x=1$. Hence the eigenvalue $\bar{C}$ is the order parameter of our group selection diffusion model. Fig. 4 offers a better view of the transition and highlights the singular nature of the segregation phase for $M=0$. Overall the effect of migration for $S>0$ is to hamper coexistence, as indicated by the need of a larger coexistence pressure to establish the coexistence regime as $M$ increases. 


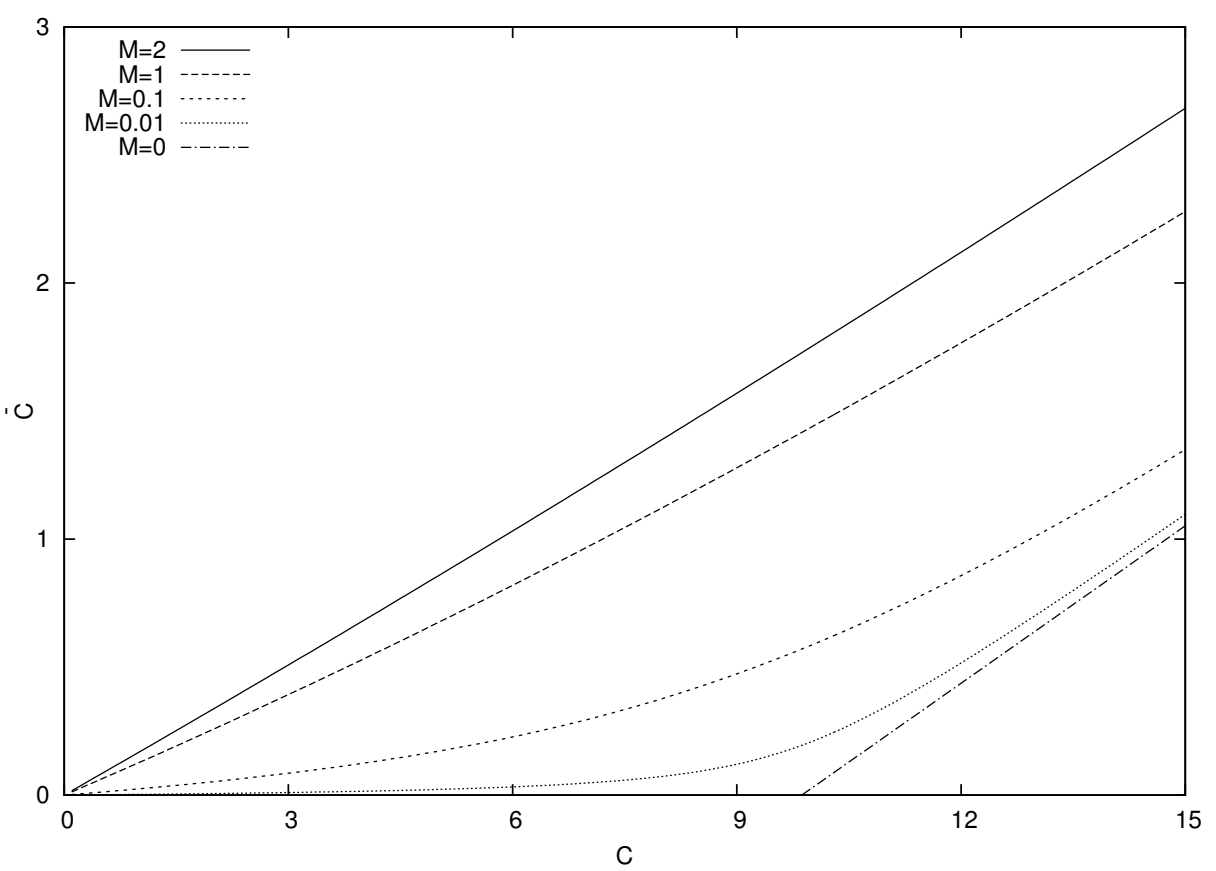

Fig. 1 Eigenvalue $\bar{C}$ of the eigenvalue problem $(18)$ as function of the coexistence pressure $C$ for the degenerate case $S=0$ and migration parameter $M$ as indicated in the figure. A phase transition takes place at $C_{c}=\pi^{2}$ in the case $M=0$.

However, the transition from $M=0$ to an arbitrarily small migration value $M \rightarrow 0$ results in a discontinuous jump on the value of the minimal coexistence pressure needed to stabilize the coexistence phase (e.g., from $C \approx 10.12$ to $C \approx 2$ for $S=1$ ). As pointed out in Sec. 3 , this is so because the $M=0$ non-ergodic segregating phase, characterized by the combination of delta functions $\phi=A_{0} \delta(x)+A_{1} \delta(x-1)$, with $A_{0}+A_{1}=1$, is unstable to the effect of migration $M>0$. In this phase, $\bar{x}=A_{1}$ depends on the initial probability density (see Appendix A) and for $\phi(x, 0)=\delta(x-1 / 2)$ we find $A_{1}=1 /[1+\exp (S / 2)]$ which is depicted in Fig. 4. In the ergodic phase (i.e., $C \geq \pi^{2}+S^{2} / 4$ ), however, the value of $\bar{x}$ at $M=0$ is approached smoothly in the limit $M \rightarrow 0$. The same is true for the order parameter $\bar{C}$ (see Fig. 3), except that in this case the behavior is continuous for all values of $C$.

\subsection{The critical line}

The critical line separates the homogeneous from the coexistence regime. Since at this line $\bar{x}=\bar{C}=0$, eq. 20 reduces to

$$
(1-x)\left[z_{c}^{\prime}+z_{c}^{2}\right]-[S(1-x)+M] z_{c}+C(1-x)=0
$$

with

$$
z_{c}(0) \equiv z_{0}=\lim _{\bar{C}, \bar{x} \rightarrow 0} \frac{\bar{C}}{M \bar{x}}
$$




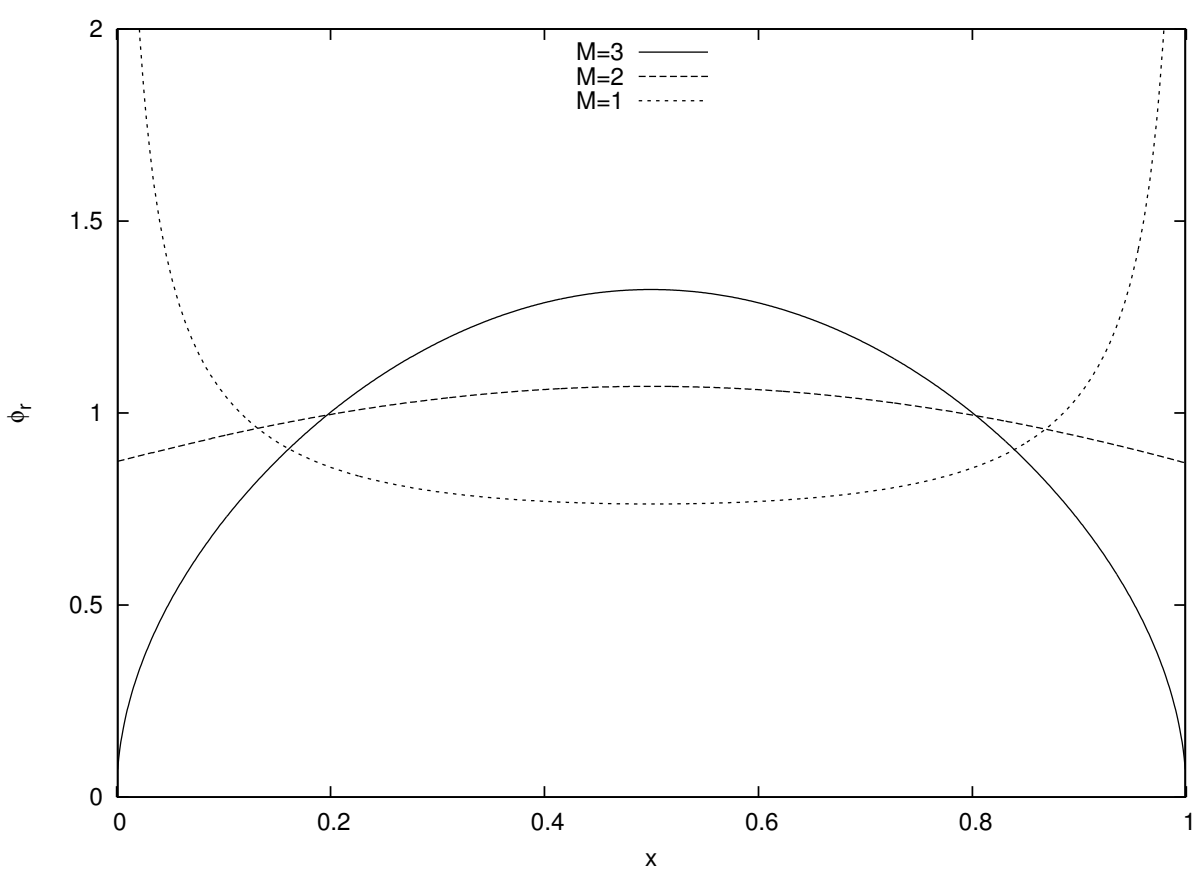

Fig. 2 Regular normalized steady-state solution $\phi_{r}$ giving the proportion of protocells that contain a fraction $x$ of type 1 templates for the degenerate case $S=0$, coexistence pressure $C=5$ and migration rates $M=3,2,1$ as indicated in the figure.

and

$$
z_{c}(1)=0 .
$$

For fixed values of the model parameters $S, C$ and $M$, eq. (25) can be solved numerically by propagating the solution from $x=1$ to $x=0$ using the Runge-Kutta algorithm. Thus, given an arbitrary set of model parameters, eq. 25 has a unique solution under condition (27), which then determines $z_{0}$ univocally. However, since eq. 25] is valid at the critical line only we need another condition to constraint the values of the model parameters. Of course, this supplementary condition is provided by eq. 26, which reads

$$
\begin{aligned}
z_{0} & =\frac{C}{M} \frac{\int_{0}^{1} d x \exp \left(-S x+y_{c}\right)(1-x)^{M}}{\int_{0}^{1} d x \exp \left(-S x+y_{c}\right)(1-x)^{M-1}} \\
& =\frac{C \int_{0}^{1} d x \exp \left(-S x+y_{c}\right)(1-x)^{M}}{1+\int_{0}^{1} d x \exp \left(-S x+y_{c}\right)(1-x)^{M}\left(-S+z_{c}\right)},
\end{aligned}
$$

where $y_{c}=\int_{0}^{x} z_{c}(\xi) d \xi$. The second line of this equation is derived from the first line by integration by parts and its sole purpose is to emphasize the fact that $z_{0}$ is finite for $M \rightarrow 0$. The limits $\bar{x} \rightarrow 0$ and $\bar{C} \rightarrow 0$ were omitted in eq. 28, so it is left implicit that this expression must be evaluated for values of $S, C$ and $M$ at the critical line. The critical line is then obtained by fixing $S$ and $M$ and adjusting $C$ such that the value of $z_{c}$ at the $x=0$ boundary of eq. 25 coincides with the value obtained using expression (28). This procedure is illustrated in Appendix B for the limit $M \rightarrow 0$, where we can obtain the analytical solution of eq. 25] as well as carry out explicitly the integrals in eq. 28. 


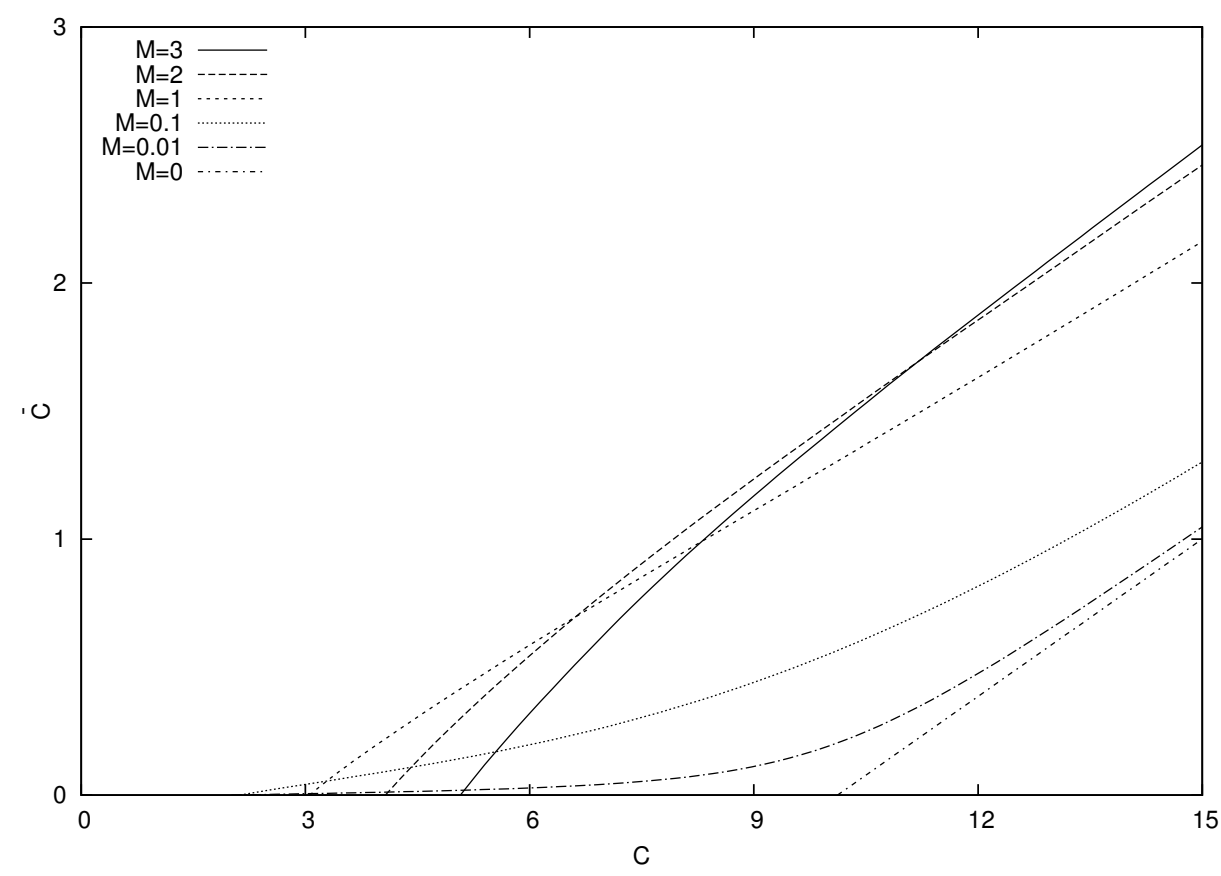

Fig. 3 Eigenvalue $\bar{C}$ of the eigenvalue problem $(18)$ as function of the coexistence pressure $C$ for a nondegenerate template competition scenario with $S=1$ and values of the migration parameter $M$ as indicated in the figure. The transition point jumps from $C_{c}=\pi^{2}+1 / 4$ for $M=0$ to $C_{c} \approx 2$ for $M \rightarrow 0$.

The final outcome of the self-consistent iterative procedure described above is summarized in Fig. 5 On the one hand, these results support the conclusion that for fixed $S>0$ increasing the migration rate $M$ hinders coexistence since it is then necessary to increase the coexistence pressure $C$ to guarantee the onset of the coexistence phase. On the other hand, a vanishingly small migration rate, represented by the curve $M \rightarrow 0$ in Fig. 5 constitutes a huge benefit to coexistence, as compared with the no-migration situation $M=0$ when the onset of the coexistence phase happens for $C>C_{c}=\pi^{2}+S^{2} / 4$ only (Fontanari and Serva. 2013). The reason is that for $M=0$ both template types are present in the population but reside in distinct protocells, and so a vanishingly small migration rate allows their meeting in a same protocell.

\subsection{Analytical approximation}

In the case $C$ and $S$ are small we can easily derive explicit expressions for the order parameter $\bar{C}$, as well as for $\bar{x}$, and so obtain an analytical expression for the critical lines shown in Fig. 5 As pointed out, the assumption that $C \ll 1$ and $S \ll 1$ amounts to saying that these two selective pressures are small with respect to random drift and migration. Considering the regular solution $\phi=\phi_{r}(x)$ of eq. (14), we begin by multiplying that equation by $x$ and then integrating over the interval $[0,1]$, yielding

$$
-S \int_{0}^{1} \phi_{r}(x) x(1-x) d x+C \int_{0}^{1} \phi_{r}(x) x^{2}(1-x) d x-\bar{x} C \int_{0}^{1} \phi_{r}(x) x(1-x) d x=0 .
$$




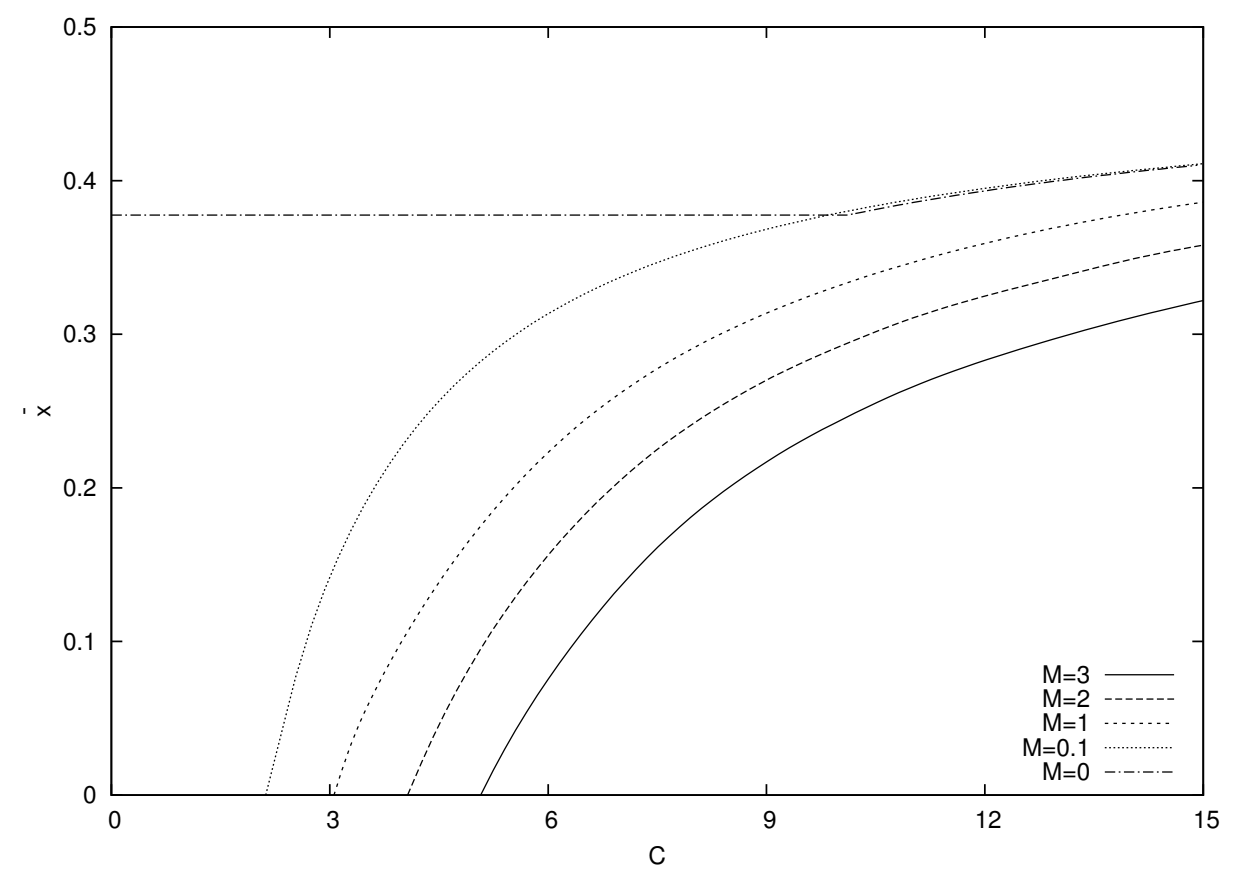

Fig. 4 Mean frequency of type 1 templates $\bar{x}$ as function of the coexistence pressure $C$ for $S=1$ and $M$ as indicated in the figure. For $M=0$, the segregating phase for $C<\pi^{2}+S^{2} / 4 \approx 10.12$ is non-ergodic and the result exhibited was obtained with the initial probability density $\phi(x, 0)=\delta(x-1 / 2)$ (see Appendix A).

Next, to obtain results that are correct to first order in $S$ and $C$, we need only to replace $\phi_{r}$ by its expression for $S=C=0$ [see eq. (17)], namely, the Beta distribution

$$
\hat{\phi}_{0}=\frac{x^{M \bar{x}-1}(1-x)^{M(1-\bar{x})-1}}{B[M \bar{x}, M(1-\bar{x})]}
$$

where $B(x, y)$ is the standard Beta function (Abramowitz and Stegun 1972). The final result is simply

$$
\bar{x}=\frac{1}{2}\left[1-(M+2) \frac{S}{C}\right],
$$

from where we get $C_{c}=(M+2) S$ which agrees with the curves shown in Fig. 5 for small $S$. For $S=0$ eq. 31 yields $\bar{x}=1 / 2$ which is actually valid for all $C$ since $\phi_{r}(x)=\phi_{r}(1-x)$ in this case.

Finally, to first order in $S$ and $C$ the order parameter $\bar{C}$ is given by

$$
\bar{C}=C \int_{0}^{1} \hat{\phi}_{0}(x) x(1-x) d x=\frac{C M}{M+1} \bar{x}(1-\bar{x})=\frac{C M}{4(M+1)}\left[1-(M+2)^{2} \frac{S^{2}}{C^{2}}\right],
$$

which fits very well the curves of Fig. 1 in the small $C$ regime, but fails to describe the results of Fig. 3 for $S=1$ since in that case the condition of small $S$ is not satisfied. 


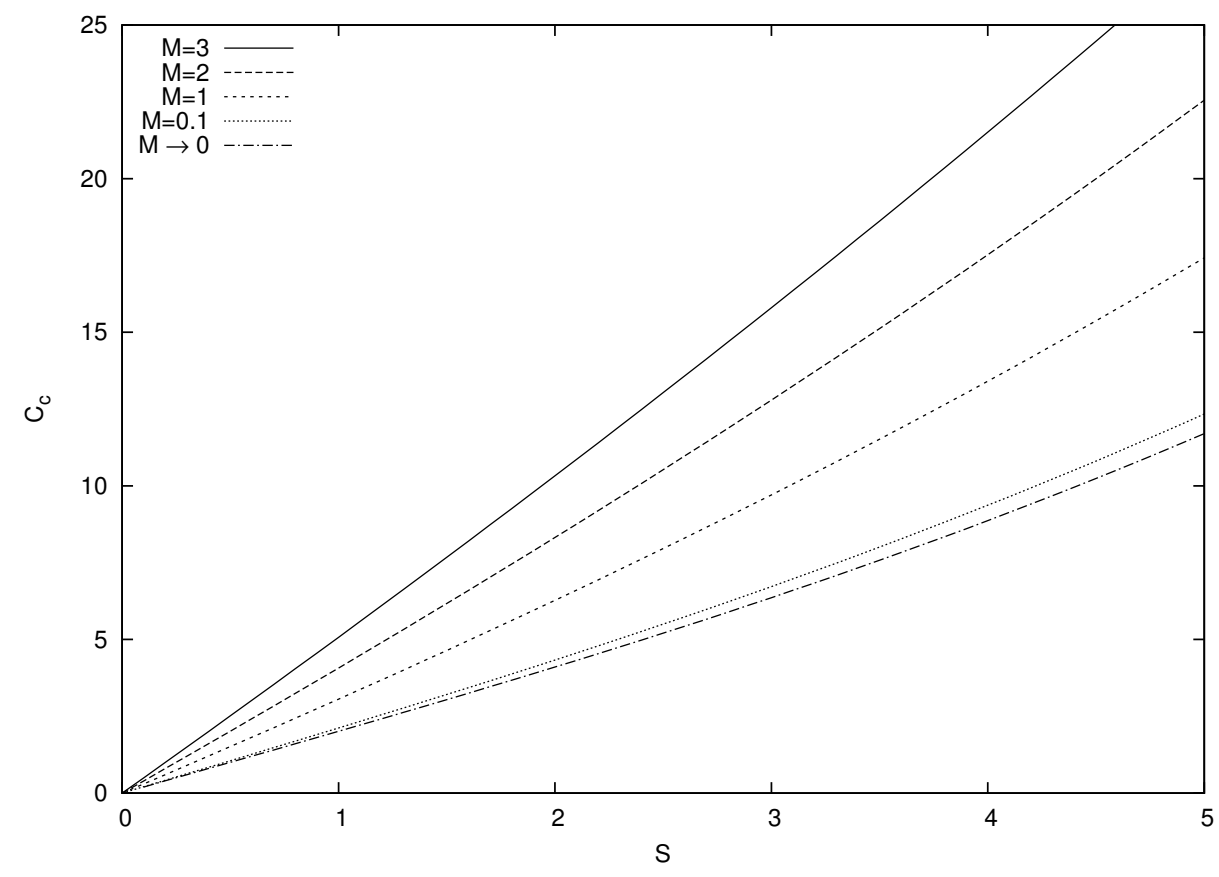

Fig. 5 Critical coexistence pressure $C_{c}$ as function of the selective advantage $S$ of type 2 templates. For fixed $M$, the lines separate the homogeneous regime $\left(C \leq C_{c}\right)$ where the population is dominated by type 2 templates from the coexistence regime $\left(C>C_{C}\right)$ where both template types cohabit the same protocell.

\subsection{Discussion}

Here we address two issues that were somewhat glossed over in the previous sections. The first issue is the difference between the limit $M \rightarrow 0$ and the case $M=0$. From the physical perspective, that difference is clear: in the absence of migration $(M=0)$ there appears a segregation phase for $C<\pi^{2}+S^{2} / 4$ which is unstable to the effect of a vanishingly small migration rate $(M \rightarrow 0)$. However, from the mathematical perspective that difference is blurred by the fact that the limit $M \rightarrow 0$ is obtained simply by setting $M=0$ in our equations. The key point here is that by writing the regular solution of eq. 14 in the form $\phi_{r}(x) \propto \phi_{0}(x) \psi(x)$ with $\phi_{0}$ and $\psi$ given by eqs. (17) and (18), respectively, we constrained the subsequent analysis to the region $M>0$ only, since in that form $\phi_{r}$ is not normalizable for $M=0$. We note that for $M=0$ the regular solution of eq. 14 , which exists for $C \geq \pi^{2}+S^{2} / 4$, is finite at the extremes $x=0$ and $x=1$ and so it is always normalizable, as expected (Fontanari and Serva, 2013). Thus setting $M=0$ in eqs. 25 and 28 actually means taking the limit $M \rightarrow 0$ of eq. (14).

The second issue concerns the uniqueness of the eigenvalue $\bar{C}$ of the second-order differential equation for $\psi$, eq. 18 . In fact, if there were no constraints on $\psi$ then there would be an infinity of admissible values for the eigenvalue $\bar{C}$ as well as for the eigenfunctions $\psi$. It is the condition that $\psi$ be positive and normalizable that reduces the acceptable solutions to a single one. We note that by writing $\psi=e^{y}$ and solving numerically for $y$ (see eq. (19)) we have automatically restricted the numerical analysis to the valid regime $\psi>0$ only. 


\section{Conclusion}

Contrary to the acrimony that has accompanied the group selection accounts of altruism and eusociality since the 1960s (Wynne-Edwards, 1962, Williams, 1966, Nowak et al., 2010, Rousset and Lion 2011), group selection ideas have been mainstream in the prebiotic evolution context (Michod, 1983, Alves et al., 2001) since there is a consensus that the compartmentalization of templates was an essential stage in the process of molecular evolution (Bresch et al. 1980, Eigen et al., 1980). In addition, compartmentalization offers a solution to the problem of the coexistence between different templates (Niesert et al. 1981; Silvestre and Fontanari, 2008), which is the topic we address in this paper. We should mention, however, that within the context of the maintenance of cooperation the group selection or, more generally, the multilevel selection approach has been applied to the study of the dynamics of cancer, which may be viewed as a result of the breakdown of cooperation between cells in the body (Michor et al. 2004, Bellomo and Delitala, 2008, Bellouquid et al., 2013).

In this contribution we build on the seminal paper by Kimura (1983), which presented a diffusion model incorporating group selection, and study a group selection pressure towards the coexistence of two types of templates that are differentiated by their replication rates. Our focus is on the effect of template swapping (migration) among protocells. This is a key process within the modern prebiotic scenario, which is based on the radical notion of an ancestral community of cell lines lacking long-term genetic history and individuality, rather than of a single ancestral organism (Woese, 1998).

We find that the progression of the template type that exhibits the selective advantage at the individual level is greatly promoted by migration, in the same manner that an antibiotic resistant gene spreads among a population comprising different bacterial species. In that sense, migration hinders coexistence. Nevertheless, migration is very effective to counterweight the homogenizing effect of random drift (i.e., the fixation of a template type) so that in the degenerate case, where there is no selective advantage at the individual level, coexistence is the only possible outcome of the evolutionary process. In addition, even in the non-degenerate case, a small amount of template swapping increases greatly the parameter range for which coexistence is stable in comparison with the case where there is no migration at all.

An interesting aspect of the diffusion model of group selection is the existence of a continuous transition between a homogeneous regime dominated by the more efficient template type and a coexistence regime where the two template types cohabit a same protocell. The order parameter that characterizes these regimes is the eigenvalue $\bar{C}$ of the eigenvalue problem (14), whose eigenfunction is the fraction of protocells with a given template composition at the the steady state. In particular, we find $\bar{C}>0$ in the coexistence regime, and $\bar{C}=0$ in the homogeneous regime with $\bar{C}$ vanishing linearly with the distance to the critical line that separates those regimes.

A simplifying feature of the model with migration is that the evolutionary dynamics is ergodic, i.e., the steady-state solution does not depend on the details of the initial distribution of templates among the protocells, provided the two template types are present in the population at the initial time. In fact, in the homogeneous phase the more efficient template type fixates in all protocells with probability one, whereas in the coexistence phase the distribution of template compositions inside the protocells are described univocally by the regular solution of eq. (14). The dynamics is non-ergodic only in the segregating phase that appears for low coexistence pressure values in the case migration is not allowed (Fontanari and Serva, 2013). For that case, we derive in Appendix A exact analytical expressions for the probability that one of the two template types fixates in a given protocell. Most interest- 
ingly, this kind of local fixation occurs both in the ergodic and in the non-ergodic phases of the model in the absence of migration and so this model offers a rare instance of subdivided population where the (local) fixation probabilities can be calculated exactly (Slatkin, 1981; Blythe, 2007).

To conclude, a word is in order about the stability of the steady-state solutions of the non-linear (and non-local) partial differential equation that determines the time evolution of the protocell population, eq. (9). On physical grounds one expects the existence of a coexistence regime for large values of the coexistence group selection pressure $C$ and so the stability of the steady-state regular solution $\phi=\phi_{r}(x)$, which satisfies eq. (14). In addition, in the absence of the coexistence pressure $(C=0)$ the only steady-state solution is the homogeneous one, i.e., $\phi=\delta(x-1)$. Whereas the regular solution exists for $C>C_{c} \approx(M+2) S$ only, the homogeneous solution exists for all $C \geq 0$ and so a possible instability of the regular solution at a finite value of $C>C_{c}$ would shift the transition point as well as turn the transition from continuous to discontinuous, in the sense that the eigenvalue $\bar{C}$ would jump to zero at the new hypothetical transition point. The analysis of the stability of the steadystate solutions by techniques such as the spectral theory in infinite dimensions (Engel and Nagel 2000) is a most interesting and challenging enterprise that could reveal the influence of the parameters $S, C$ and $M$ on the relaxation time to equilibrium as well as confirm the steady-state prediction of the critical point $C_{c}$ separating the homogeneous and coexistence regimes. We hope our paper will motivate further studies on this research line.

\section{Appendix A: Local fixation probability for the $M=0$ non-ergodic segregation regime}

As shown by Fontanari and Serva (2013), setting $M=0$ in eq. (14) yields two possible steady-state solutions: the solution corresponding to the ergodic coexistence phase, which is a combination of two Delta functions and a regular function, $\phi(x)=A_{0} \delta(x)+A_{1} \delta(x-1)+$ $B \phi_{r}(x)$, with $A_{0}+A_{1}+B=1$, and the solution corresponding to the non-ergodic segregation phase, which is a combination of the two Delta functions, $\phi(x)=A_{0} \delta(x)+A_{1} \delta(x-1)$, with $A_{0}+A_{1}=1$. The non-ergodic regime, which is our focus here, occurs for $C<\pi^{2}+S^{2} / 4$. Note that in both regimes $A_{1}$ may be interpreted as the probability that the type 1 template fixates in a given protocell and a similar interpretation holds for $A_{0}$ as well. However, the result $\bar{x}=A_{1}$, which we used to draw the curve for $M=0$ in fig. 4, holds in the segregation regime only. In Fontanari and Serva (2013) we have calculated the dependence of the weight $A_{1}$ on the initial probability density $\phi(x, 0)$ for $S=0$ only, and in this appendix we generalize that calculation for $S \geq 0$.

We begin by rewriting eq. 97 for $M=0$,

$$
\frac{\partial}{\partial \tau} \phi(x, \tau)=\frac{\partial^{2}}{\partial x^{2}}[x(1-x) \phi(x, \tau)]+S \frac{\partial}{\partial x}[x(1-x) \phi(x, \tau)]+[C x(1-x)-\bar{C}(\tau)] \phi(x, \tau)
$$

and introducing the abbreviation $\langle f(x)\rangle_{\tau}=\int_{0}^{1} f(x) \phi(x, \tau) d x$ for the expected value of a regular function $f(x)$ at time $\tau$. Hence

$$
\begin{aligned}
\frac{d}{d \tau}\langle f(x)\rangle_{\tau}= & \left\langle x(1-x) \frac{\partial^{2} f(x)}{\partial x^{2}}\right\rangle_{\tau}-S\left\langle x(1-x) \frac{\partial f(x)}{\partial x}\right\rangle_{\tau} \\
& +C\langle x(1-x) f(x)\rangle_{\tau}-\bar{C}(\tau)\langle f(x)\rangle_{\tau}
\end{aligned}
$$

with $\bar{C}(\tau)=C\langle x(1-x)\rangle_{\tau}$. The idea is to choose a function $f(x)$ such that the first three terms of the right hand side of eq. (34) cancel out. This choice depends on the value of the parameter $\Gamma \equiv C-S^{2} / 4$ as discussed next. We note that $\Gamma<\pi^{2}$ in the non-ergodic regime. 
Region $0<\Gamma<\pi^{2}$. In this region we choose $f(x)=e^{S x / 2} \sin (\sqrt{\Gamma} x+\theta)$ where $\theta$ is an arbitrary constant. Then eq. (34) rewrites

$$
\frac{d}{d \tau}\left\langle e^{S x / 2} \sin (\sqrt{\Gamma} x+\theta)\right\rangle_{\tau}=-\bar{C}(\tau)\left\langle e^{S x / 2} \sin (\sqrt{\Gamma} x+\theta)\right\rangle_{\tau}
$$

which has the formal solution

$$
\frac{\left\langle e^{S x / 2} \sin (\sqrt{\Gamma} x+\theta)\right\rangle_{\tau}}{\left\langle e^{S x / 2} \sin (\sqrt{\Gamma} x+\theta)\right\rangle_{0}}=\exp \left[-\int_{0}^{\tau} \bar{C}(\eta) d \eta\right] .
$$

As the right hand side of this equation does not depend on $\theta$, neither does the ratio in its left hand side. Hence, equating the ratios evaluated at $\theta=0$ and $\theta=\pi / 2-\sqrt{\Gamma} / 2$ yields

$$
\frac{\left\langle e^{S x / 2} \sin (\sqrt{\Gamma} x)\right\rangle_{\tau}}{\left\langle e^{S x / 2} \cos [\sqrt{\Gamma}(x-1 / 2)]\right\rangle_{\tau}}=\frac{\left\langle e^{S x / 2} \sin (\sqrt{\Gamma} x)\right\rangle_{0}}{\left\langle e^{S x / 2} \cos [\sqrt{\Gamma}(x-1 / 2)]\right\rangle_{0}}
$$

In the limit $\tau \rightarrow \infty$ we have

$$
\left\langle e^{S x / 2} \sin (\sqrt{\Gamma} x)\right\rangle_{\infty}=A_{1} e^{S / 2} \sin (\sqrt{\Gamma})
$$

and

$$
\begin{aligned}
\left\langle e^{S x / 2} \cos [\sqrt{\Gamma}(x-1 / 2)]\right\rangle_{\infty} & =\left(A_{0}+A_{1} e^{S / 2}\right) \cos (\sqrt{\Gamma} / 2) \\
& =\left[1+A_{1}\left(e^{S / 2}-1\right)\right] \cos (\sqrt{\Gamma} / 2)
\end{aligned}
$$

which leads to

$$
A_{1}=\frac{1}{1+e^{S / 2}\left(\Xi_{0}-1\right)}
$$

where

$$
\Xi_{0}=2 \sin (\sqrt{\Gamma} / 2) \frac{\left\langle e^{S x / 2} \cos [\sqrt{\Gamma}(x-1 / 2)]\right\rangle_{0}}{\left\langle e^{S x / 2} \sin (\sqrt{\Gamma} x)\right\rangle_{0}}
$$

In the limit $\Gamma \rightarrow \pi^{2}$, we have $\Xi_{0} \rightarrow 2$ regardless of the initial probability density $\phi(x, 0)$ and so $A_{1} \rightarrow A_{1}^{c}=1 /\left(1+e^{S / 2}\right)$. In addition, for the initial probability density $\phi(x, 0)=$ $\delta(x-1 / 2)$ used to calculate $\bar{x}$ at $M=0$ in Fig. 4, the dependence on $\Gamma$ (and hence on $C$ ) disappears and so $A_{1}=A_{1}^{c}$.

Region $-S^{2} / 4<\Gamma<0$. In this region the choice $f(x)=e^{S x / 2}\left(e^{u x}+\theta e^{-u x}\right)$ with $u=\sqrt{-\Gamma}$ and $\theta$ arbitrary leads to the canceling of the first three terms of the right hand side of eq. (34) yielding

$$
\frac{\left\langle e^{S x / 2}\left(e^{u x}+\theta e^{-u x}\right)\right\rangle_{\tau}}{\left\langle e^{S x / 2}\left(e^{u x}+\theta e^{-u x}\right)\right\rangle_{0}}=\exp \left[-\int_{0}^{\tau} \bar{C}(\eta) d \eta\right] .
$$

The same argument used in the analysis of the $\Gamma>0$ region allows us to equate the ratio that appear in the left hand side of this equation for $\theta=-1$ and $\theta=0$,

$$
\frac{\left\langle e^{S x / 2} \sinh (u x)\right\rangle_{\tau}}{\left\langle e^{S x / 2+u x}\right\rangle_{\tau}}=\frac{\left\langle e^{S x / 2} \sinh (u x)\right\rangle_{0}}{\left\langle e^{S x / 2+u x}\right\rangle_{0}} .
$$


Finally, taking the limit $\tau \rightarrow \infty$ yields

$$
A_{1}=\frac{1}{1+e^{S / 2}\left[\Omega_{0} \sinh (u)-e^{u}\right]}
$$

where

$$
\Omega_{0}=\frac{\left\langle e^{S x / 2+u x}\right\rangle_{0}}{\left\langle e^{S x / 2} \sinh (u x)\right\rangle_{0}} .
$$

By taking the limit $u \rightarrow 0$ we can easily verify that $A_{1}$ is continuous at the boundary of the two regions. In addition, in the limit $C \rightarrow 0$, i.e., $u \rightarrow S / 2$ we recover the classical formula for the fixation of an allele with selective disadvantage $S$ (Crow and Kimura. 1970),

$$
A_{1}=\frac{\left\langle e^{S x}\right\rangle_{0}-1}{e^{S}-1} .
$$

Similarly to our finding in the analysis of the previous region, the initial probability density $\phi(x, 0)=\delta(x-1 / 2)$ yields $A_{1}=1 /\left(1+e^{S / 2}\right)$ regardless of the value of $C$, as shown in Fig. 4

\section{Appendix B: Critical line for the limit $M \rightarrow 0$}

Setting $M=0$ in eq. (25) yields

$$
z_{c}^{\prime}+z_{c}^{2}-S z_{c}+C=0
$$

with the condition

$$
z_{c}(1)=0 .
$$

In the region $C-S^{2} / 4>0$ its solution is

$$
z_{c}(x)=\frac{S}{2}-\gamma \tan (\gamma x+\theta)
$$

where $\gamma=\sqrt{C-S^{2} / 4}$ and $\theta=\theta(\gamma, S)$ is fixed by condition 48 as

$$
\frac{S}{2}-\gamma \tan (\gamma+\theta)=0
$$

We note that the critical value $C_{c}(S)$ is in the region $C-S^{2} / 4>0$ (see Fig. 5). To evaluate eq. 28. we use the equality

$$
\exp \left(-S x+y_{c}\right)=\frac{\cos (\gamma x+\theta)}{\cos (\theta)} \exp (-S x / 2) .
$$

which follows directly from the definition $y_{c}(x)=\int_{0}^{x} z_{c}(\xi) d \xi$ with $z_{c}$ given by 49 . Now the integrals in eq. 28, can be readily evaluated yielding

$$
\frac{S}{2}-\gamma \tan (\theta)=\frac{\gamma\left[e^{-\frac{S}{2}} \sin (\gamma+\theta)-\sin (\theta)\right]-\frac{S}{2}\left[e^{-\frac{S}{2}} \cos (\gamma+\theta)-\cos (\theta)\right]}{e^{-\frac{S}{2}} \cos (\gamma+\theta)} .
$$


This equation can be further simplified using the equalities $\sin (\gamma+\theta)=S /(2 \sqrt{C})$ and $\cos (\gamma+\theta)=\gamma / \sqrt{C}$ that follow from eq. 50. The final result is simply

$$
\gamma=\sqrt{C} e^{\frac{S}{2}} \cos (\theta)
$$

Finally, we rewrite eq. 50 as

$$
\theta=\arctan \left(\frac{S}{2 \gamma}\right)-\gamma
$$

in order to make clear that eq. 53 yields a relation $C=C_{c}(S)$, which is the critical line $M \rightarrow 0$ depicted in Fig.5.

Acknowledgements The research of J.F.F. was supported in part by Conselho Nacional de Desenvolvimento Científico e Tecnológico (CNPq) and the research of M.S. was partially supported by PRIN 2009 protocollo n.2009TA2595.02.

\section{References}

Abramowitz, M., \& Stegun, I.A. (1972). Handbook of Mathematical Functions with Formulas, Graphs, and Mathematical Tables. New York: Dover Publications.

Alves, D., Campos, P.R.A., Silva, A.T.C., \& Fontanari, J.F. (2001). Group selection models in prebiotic evolution. Phys. Rev. E, 63, 011911.

Aoki, K. (1982). A condition for group selection to prevail over counteracting individual selection. Evolution, 36, 832-842.

Bellomo, N., \& Delitala, M. (2008). From the mathematical kinetic and stochastic game theory to modelling mutations, onset, progression and immune competition of cancer cells. Phys. Life Rev., 5, 183-206.

Bellouquid, A., de Angelis, E., \& Knopoff, D. (2013). From the modeling of the immune hallmarks of cancer to a black swan in biology. Math. Models Methods Appl. Sci., 23, 949-978.

Bresch, C., Niesert, U., \& Harnasch, D. (1980). Hypecycles, Parasites and Packages. J. Theor. Biol., 85, 399-405.

Blythe, R.A. (2007). The propagation of a cultural or biological trait by neutral genetic drift in a subdivided population. Theor. Pop. Biol., 71, 454-472.

Chalub, F.A.C.C., \& Souza, M.O. (2009). A non-standard evolution problem arising in population genetics. Commun. Math. Sci., 7, 489-502.

Crow, J. F., \& Kimura, M. (1970). An introduction to population genetics theory. New York: Harper \& Row.

Czárán, T., \& Szathmáry, E. (2000). Coexistence of replicators in prebiotic evolution. In: Dieckmann, U., Law, R., Metz, J.A.J. (Eds.), The Geometry of Ecological Interactions: Simplifying Spatial Complexity. Cambridge, UK, Cambridge University Press, pp. 116135.

Donato, R., Peliti, L., \& Serva, M. (1997). The selection of altruistic behaviour. Theor. Biosci., 116, 309-320.

Doolittle, W.F. (2000). Uprooting the Tree of Life. Sci. Am., 282 , 90-95.

Eigen, M. (1971). Self-organization of matter and the evolution of macromolecules. Naturwiss., 58, 465-523. 
Eigen, M., \& Schuster, P. (1978). The Hypercycle. A Principle of Natural Self-Organization. Part C: The Realistic Hypercycle. Naturwiss., 65, 341-369.

Eigen, M., Gardiner Jr., W.C.. \& Schuster, P. (1980). Hypercycles and Compartments. J. Theor. Biol., 85, 407-411.

Engel, K.-J., \& Nagel, R. (2000). One-parameter semigroups for linear evolution equations. New York: Springer-Verlag.

Eshel, I. (1972). On the neighbor effect and the evolution of altruistic traits. Theor. Pop. Biol., 3, 258-277.

Fontanari, J. F., Santos, M., \& Szathmáry, E. (2006). Coexistence and error propagation in pre-biotic vesicle models: A group selection approach. J. Theor. Biol., 239, 247-256.

Fontanari, J. F., \& Serva, M. (2013). Solvable model for template coexistence in protocells. Europhys. Lett., 101, 38006.

Fontanari, J. F., \& Serva, M. (2014). Nonlinear group survival in Kimura's model for the evolution of altruism. Math. Biosc., 249, 18-26.

Kimura, M. (1983). Diffusion model of intergroup selection, with special reference to evolution of an altruistic character. Proc. Natl. Acad. Sci. USA, 80, 6317-6321.

Manrubia, S. C., \& Briones, C. (2007). Modular evolution and increase of functional complexity in replicating RNA molecules. $R N A, 13,97-107$.

Maynard Smith, J., \& Szathmáry, E. (1995). The Major Transitions in Evolution. Oxford: Freeman \& Co.

Mayr, E. (2001). The philosophical foundation of Darwinism. P. Am. Philos. Soc., 145, 488495.

Mayr, E. (2002). What evolution is. New York: Basic Books.

Mayr, E. (2004). 80 years of watching the evolutionary scenery. Science, 305, 46-47.

Michod, R.E. (1983). Population biology of the first replicators: on the origin of genotype, phenotype, and organism. Am. Zool., 23, 5-14.

Michor, F., Iwasa, Y., \& Nowak, M. A. (2004). Dynamics of cancer progression. Nat. Rev. Cancer, 4, 197-205.

Niesert, U., Harnasch, D., \& Bresch, C. (1981). Origin of life between Scylla and Charybdis. J. Mol. Evol.,17, 348-353.

Nowak, M. A., \& Sigmund, K. (2004). Evolutionary dynamics of biological games. Science, 303, 793-799.

Nowak, M. A., Tarnita, C. E., \& Wilson, E. O. (2010). The evolution of eusociality Nature, 466,1057-1062.

Ogura, Y., \& Shimakura, N. (1987). Stationary solutions and their stability for Kimura's diffusion model with intergroup selection. J. Math. Kyoto Univ., 27, 305-347.

Press, W. H., Teukolsky, S. A., Vetterling, W. T., \& Flannery, B. P. (1992). Numerical Recipes in Fortran: The Art of Scientific Computing. Cambridge, MA: Cambridge University Press.

Rousset, F., \& Lion, S. (2011). Much ado about nothing: Nowak et al.'s charge against inclusive fitness theory. J. Evol. Biol., 24,1386-1392.

Silvestre, D. A. M. M., \& Fontanari, J. F. (2008). Package models and the information crisis of prebiotic evolution. J. Theor. Biol., 252, 326-337.

Slatkin, M. (1981). Fixation probabilities and fixation times in a subdivided population. Evolution, 35, 477-488.

Szathmáry, E., \& Demeter, L. (1987). Group selection of early replicators and the origin of life. J. Theor. Biol.,128, 463-486.

Wright, S. (1951). The genetical structure of populations. Ann. Eugen., 15 323-354. 
Williams, G. C. (1966). Adaptation and Natural Selection: A Critique of Some Current Evolutionary Thought. Princeton: Princeton University Press.

Woese, C. (1998). The Universal Ancestor. Proc. Natl. Acad. Sci. USA, 95, 6854- 6859.

Wynne-Edwards, V. C. (1962). Animal Dispersion in Relation to Social Behavior. London: Oliver \& Boyd. 